\title{
Patients' interpretation of symptoms as a cause of delay in reaching hospital during acute myocardial infarction
}

\author{
R Horne, D James, K Petrie, J Weinman, R Vincent
}

\begin{abstract}
Objective-To examine whether the association between expected symptoms of acute myocardial infarction and actual symptoms predicted delay in reaching hospital and help seeking behaviour.

Design-During hospital convalescence, participants completed a structured interview designed to measure symptom experience and help seeking behaviour following the onset of symptoms of acute myocardial infarction.

Patients-88 patients admitted to hospital with their first myocardial infarction

Main outcome measures-Delay in reaching hospital from onset of worst symptoms, obtained from ambulance and hospital records.

Results-The most common symptoms expected by patients with myocardial infarction were central chest pain $(76 \%)$, radiating arm or shoulder pain $(34 \%)$, and collapse $(26 \%)$. The most common symptoms experienced were sweats or feeling feverish $(78 \%)$, chest pain $(64 \%)$, and arm, shoulder, or radiating pain (66\%). A mismatch between symptoms experienced and those expected occurred in $58 \%$ of patients, and was associated with delay. Patients who experienced a mismatch between expectation and actual symptoms also were more likely to have a third party
\end{abstract} decide to call for help.

Conclusions-The experience and interpretation of symptoms is an important source of delay and help seeking following onset of myocardial infarction symptoms.

(Heart 2000;83:388-393)

Keywords: myocardial infarction; symptoms; delayed diagnosis

Centre for Health Care Research, University of Brighton, Falmer Campus, Brighton BN1 9PH, UK

R Horne

School of Pharmacy and Biomolecular Sciences, University of Brighton, Brighton, UK

D James

Department of Behavioural Medicine, University of Auckland, Auckland, New Zealand

K Petrie

Division of Psychiatry and Psychology, Guy's, King's and St Thomas' Medical Schools (Guy's Campus), London SE1, UK

J Weinman

Cardiology, Brighton Health Care NHS Trust, Royal Sussex County Hospital, Brighton, UK $\mathrm{R}$ Vincent

Correspondence to: Dr Horne

email:

r.horne@brighton.ac.uk

Accepted 23 November 1999
The efficacy of thrombolysis in reducing morbidity and mortality from myocardial infarction is well established. ${ }^{1}$ However, the effectiveness of this treatment is dependent on administration of the thrombolytic agent soon after the onset of symptoms, and it is likely that reducing the delay in reaching hospital after acute myocardial infarction would improve survival. ${ }^{23}$

The main cause of delay in patients reaching hospital after myocardial infarction is the time taken for the patient to decide that their symptoms need medical attention (patient decision time). ${ }^{45}$ Previous research on patient delay following the onset of symptoms in myocardial infarction has largely focused on demographic, symptom related, and clinical factors as predictors of delay in reaching hospital..$^{6-8}$ This research has produced a conflicting picture of the patient who delays. For example, older patients (more than 55 years of age), women, and patients of lower socioeconomic status had longer delays in some studies but not others. ${ }^{9}$ Other studies have focused on symptom experience as a source of delay. Delay does not appear to be related to the severity of pain associated with the acute event. ${ }^{10}{ }^{11}$ However, in one study the strongest predictor of early arrival in hospital was the belief that the symptoms were those of a heart attack. ${ }^{12}$ Similarly, a recent qualitative study suggested that patients who sought help early knew a wider range of symptoms of a heart attack. ${ }^{13}$ The notion that patients may delay because they do not recognise their symptoms has led to increasing interest in the role of symptom interpretation as a source of delay in acute myocardial infarction. ${ }^{514}$

There is growing evidence from other illnesses to show that patients' interpretation of their symptoms can influence help seeking behaviour. ${ }^{15-17}$ Previous work has shown that people have pre-existing ideas of the symptoms associated with common illnesses and that they identify diseases by comparing the symptoms they experience with those they expect for a given disease. ${ }^{18}$ The extent to which the symptoms experienced during acute myocardial infarction match prior expectations has yet to be investigated, but recognising the symptoms as those of a heart attack may have important implications for the time taken to obtain medical treatment.

It is proposed that decisions about what action to take in response to the symptoms of acute myocardial infarction are influenced by patients' pre-existing ideas about heart attacks and the extent to which their symptom experience matches their expectations. However, little is currently known about this process. We hypothesise that the more the symptoms experienced (for example, chest pain with radiating arm pain) match those expected of a heart attack, the more likely it is that patients will seek treatment promptly. However, not all infarcts present with typical chest pain, and various other symptoms may be present which could prevent the patient from suspecting a heart attack. Alternatively, patients may have 
an inaccurate model of the symptoms that are part of myocardial infarction and so fail to recognise the seriousness of their condition. Both situations cause delay. In this study we investigated the relation between symptom experience, symptom expectation, and delay in reaching hospital. The aims of the study were to assess the extent to which the symptoms experienced by the patient matched their prior expectations of what a heart attack would be like, and to quantify the effect of mismatch on help seeking behaviour and delay in reaching hospital.

\section{Methods}

PARTICIPANTS

A systematic sample (every third patient) of patients with their first myocardial infarction was recruited from the coronary care unit of the Royal Sussex County Hospital, Brighton. Patients with acute myocardial infarction are always admitted through the accident and emergency department (A\&E). The $A \& E$ records were checked on a daily basis and every patient admitted with chest pain to the coronary care unit (CCU) or the medical wards was followed up for further evaluation. With ethics committee approval, every third patient eligible for study was approached by the researcher and invited to take part. Patients were eligible for inclusion if they had an unequivocal diagnosis of first myocardial infarction and if the acute event occurred outside the hospital. Patients were not eligible for inclusion if they could not understand enough English to complete the interview schedule, and if they were transferred to other centres (for example, for emergency surgery) within three days of admission. In order to allow time for convalescence, patients were approached no sooner than three days after admission.

Nine patients who fulfilled the entry criteria refused to participate, and 88 patients completed the interview. The mean (SD) age of the sample was 61 (9.8) years and 68 patients $(77 \%)$ were male. All but one of the patients were of north European ethnic origin. Seventy seven patients $(89.5 \%)$ had been educated to secondary level, six (7\%) to tertiary level, and three $(3.5 \%)$ to higher level. Sixty six patients $(75 \%)$ were at home when the symptoms first started and $12(14 \%)$ lived alone. Over half the subjects studied $(54 \%)$ were current smokers. Twenty patients $(22 \%)$ were classified according to the Registrar General's classification for social class as socioeconomic group I and II (professional and management), 63 (72\%) were class III, IV, or V (skilled or semiskilled), and five $(6 \%)$ were unemployed (class VI). Twelve patients $(14 \%)$ had a previous diagnosis of angina.

\section{MEASURES}

A structured interview schedule was developed to elicit and score the patients' experience and interpretation of the symptoms that led to admission with acute myocardial infarction, and their referral behaviour. The schedule was developed during a previous pilot study involving 15 patients who had survived a first myocardial infarction, and was adapted from a validated methodology for eliciting and scoring symptom reports. ${ }^{19}$

\section{Experience of symptoms}

Patients indicated whether they had experienced each of a list of nine symptoms: central chest pain, chest discomfort, radiating pain or numbness (arm, shoulder, neck, or jaw), feeling dizzy or light headed, loss of consciousness, nausea or vomiting, fever or sweating, shortness of breath, and any other symptom or pain. Chest discomfort was included as a separate category to chest pain because in the pilot study several patients stressed that they experienced a "discomfort" or "tightness" in the chest region that they distinguished from chest pain. This was elicited in the interview schedule by first asking the patient if they had experienced chest pain. If they said no, they were asked if they had experienced chest tightness or discomfort.

The symptoms were categorised into two groups: "typical" and "atypical." The typical symptom group comprised those symptoms that are commonly perceived to be associated with cardiac problems. These were chest pain, radiating pain or numbness (arm, jaw, neck or shoulder), and collapse. "Collapse" was used as an umbrella term to describe fainting, loss of consciousness, and cardiac arrest. The atypical symptom group comprised other symptoms that may occur during an acute cardiac event but which are rarely highlighted in educational campaigns to raise public awareness ${ }^{13}$ and that patients may be less likely to associate with a cardiac origin. These were shortness of breath, nausea or vomiting, feeling faint or light headed, and sweats/"fever". Several patients who reported these symptoms described them as "flu-like".

\section{Severity of pain}

Patients were asked to rate the severity of the pain or discomfort they experienced on a visual analogue scale (VAS). Patients were shown the VAS and asked to record their rating using the following phrase: "On a scale of 0 to 10 (where 0 is no pain and 10 is pain as bad as you can imagine), how would you rate the severity of the pain or discomfort when it was at its worst?"

\section{Experience of prodromal symptoms}

Patients were asked if they had experienced any symptoms in the days leading up to their heart attack. Patient symptom reports were noted verbatim and categorised as "cardiac related prodromal" if they included breathlessness, chest pain, chest discomfort, radiating pain or numbness, or angina.

Prior expectations of symptoms

Patients were asked the following question: "If I had stopped you in the street before this heart attack and asked you what you thought the symptoms of a heart attack would be like, what would you have said?" Each symptom reported by the patient was recorded verbatim by the researcher. 
Perceived match between expectations and experience of symptoms

Patients who reported having prior expectations of symptoms were asked to state whether their experience of a heart attack was the same, similar to, or different from what they expected.

\section{Seeking medical help}

Two aspects of referral behaviour were elicited. First, whether the patient or someone else had called for medical help. Second, we asked how help had been sought initially. Responses were grouped into two categories: "contacted the emergency services" and "other" (for example, telephoned general practitioner, drove to hospital).

Delay

The times of onset of symptoms of acute myocardial infarction and arrival at hospital were noted from the ambulance record sheet filed in the medical notes. For those who had not come to hospital by ambulance, delay time was estimated from the onset of symptoms noted by the admitting doctor and from the time of registration in hospital noted in the $\mathrm{A} \& \mathrm{E}$ records. Delay was calculated as the time from onset of symptoms to arrival in hospital.

DATA ANALYSIS

Statistical analyses were carried out with the SPSS for Windows statistical software package. Differences in mean delay times between patients who experienced chest pain during the acute event and those who did not, and between those whose symptom experience matched expectations, were assessed using the independent $t$ test with Levene's test for equality of means. Relations between dichotomous variables (for example, the presence or absence of chest pain, or match $v$ mismatch between symptom experience and expectations) were explored using $\chi^{2}$ tests with continuity correction. Pearson correlation (two tailed) was used to investigate relations between delay time and VAS ratings of the severity of pain experienced during the acute event. Statistical significance was set at the probability level of $<0.05$. The distribution of the delay variable was found to be skewed and was therefore transformed by a $\log$ transformation. However, for ease of interpretation the results are presented for the untransformed variable (that is, the delay in hours from experience of symptoms to arrival in hospital).

\section{Results}

DELAY AND HELP SEEKING BEHAVIOUR

The mean (SD) delay between onset of symptoms and arrival in hospital was 7.3 (14.2) hours. The data were negatively skewed, in common with other studies, ${ }^{9}$ with a median delay time of 2.2 hours. Thirty nine patients ( $44 \%$ of the sample) reported that the decision to call for medical help was made by someone else present at the time of the infarct, and not by the patient themselves. In most cases (62 patients, $72 \%$ ), medical help was sought by telephoning the general practitioner or by driv- ing or taking public transport to the hospital rather than by dialling the emergency services as recommended..$^{20}$ Failure to call the emergency services was associated with significantly longer delay times (mean $1=9.67$ hours $v$ mean $2=1.65$ hours; $t=2.50 ; \quad \mathrm{df}=83$; $\mathrm{p}<0.01)$. Delay was not related to age, sex, social class, or educational experience.

SYMPTOM EXPECTATIONS AND EXPERIENCE

Patients experienced a mean of 4.75 (1.74) symptoms as part of their heart attack. Fifty six patients ( $64 \%$ of the sample) reported experiencing chest pain, and $58(66 \%)$ reported arm, shoulder, or radiating pain; 37 patients (42\%) experienced both chest and arm/shoulder pain during the acute event. Twenty four patients $(27 \%)$ who did not report chest pain said that they experienced a discomfort or "tightening" in the chest area which they distinguished from chest pain. Only 14 patients $(16 \%)$ fainted or collapsed. The most common symptoms experienced were sweats or feeling "feverish", with 69 patients $(78 \%)$ using one or more of these terms to describe their symptoms.

Patients' reports of the type of symptoms they expected of a heart attack all fell into the nine categories used to elicit symptom experiences. The most common symptom expectations were of central chest pain (76\%), radiating pain across the arm, neck, or shoulder $(34 \%)$, and collapse (26\%). In contrast, few patients expected to experience the "atypical" symptoms of shortness of breath $(7 \%)$, nausea or vomiting $(3 \%)$, dizziness $(3 \%)$, or fever $(3 \%)$.

The frequency distribution of symptom experiences versus symptom expectations for typical and atypical symptom categories is shown in fig 1 . It can be seen that the degree to which symptom experiences matched expectations differed greatly between the typical and the atypical symptom groups. Most patients $(76 \%)$ expected that central chest pain would be part of a heart attack, and $56(66 \%)$ experienced this symptom. Although more patients experienced radiating pain than had expected it (34\% expected radiating pain and $66 \%$ experienced it), the contrast between expectations and experience was greatest for the atypical symptom categories of shortness of breath, nausea/vomiting, dizziness, and sweating/fever, as shown in fig 1 , right panel. Only 12 patients (13\%) expected to experience one or more of these symptoms as part of a heart attack. However, $82(93 \%)$ experienced one or more of the symptoms during the acute event.

Fifty one patients $(58 \%)$ reported a definite mismatch between symptom experiences and expectations, $29.5 \%$ reported that experiences matched expectations, and $12.5 \%$ said that they did not have a clear idea of what a heart attack would be like.

Fifty one patients (58\%) also reported that they had experienced "cardiac related" prodromal symptoms in the days leading up to their heart attack. 

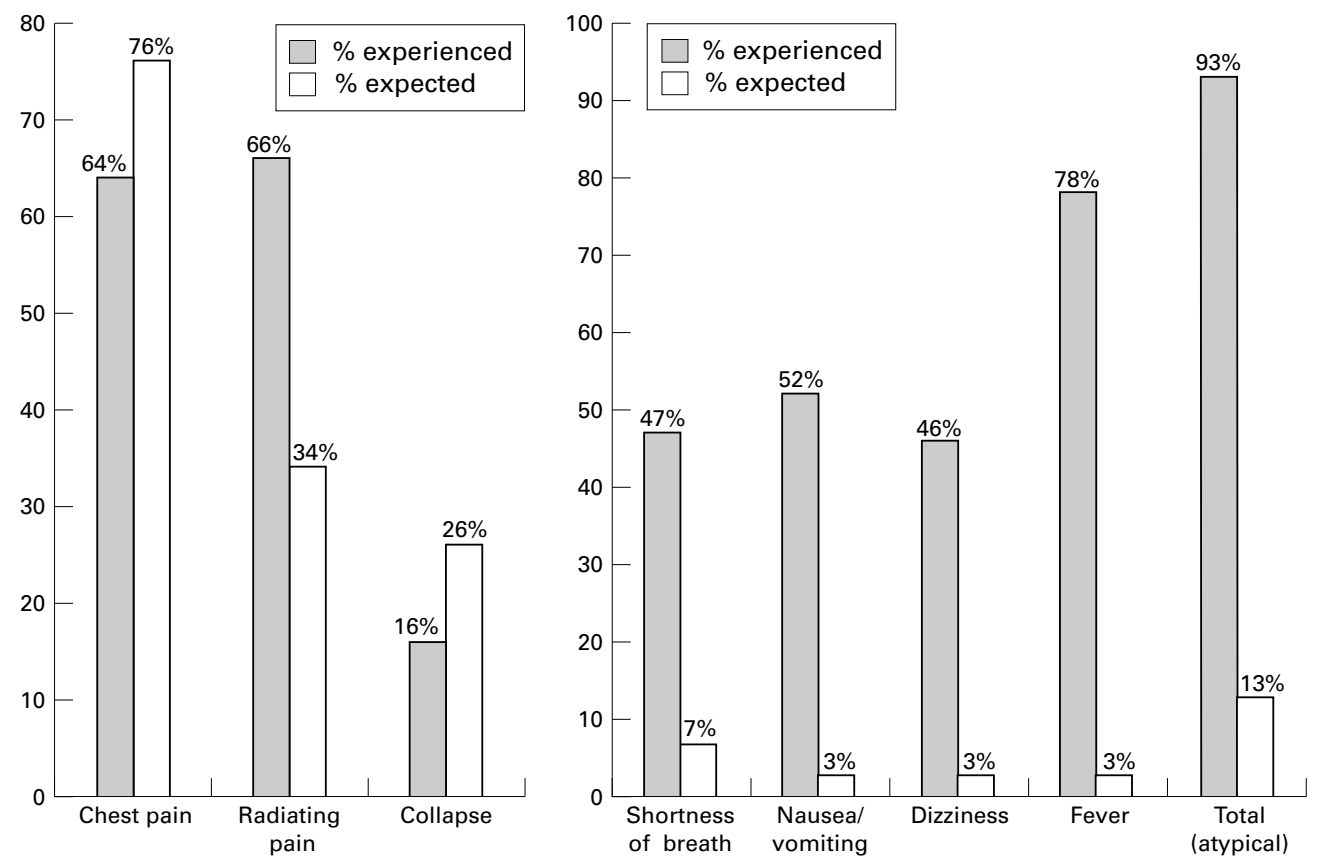

Figure 1 Left panel: expectations and experience of "typical" cardiac symptoms. Right panel: expectations and experiences of "atypical" symptoms.

SYMPTOM PERCEPTIONS AND DELAY

Patients who did not experience chest pain during the acute event had significantly longer delay times than those who did (mean $1=11.5$ hours $v$ mean $2=4.8$ hours; $t=2.2 ; \mathrm{df}=87$; $\mathrm{p}<0.05)$. The number of symptoms experienced was not in itself related to delay time. However, delay times were significantly reduced if the additional symptoms experienced were those that are commonly perceived to be typical of a heart attack. This trend can be seen in table 1 , which shows the mean delay times for those experiencing chest pain and other "typical" cardiac symptoms. A weak negative correlation was found between the patients' rating of the overall severity of the pain they experienced during the acute event and delay time (Pearson correlation, two tailed; $r=-0.24 ; \mathrm{p}<0.05)$, with more severe pain predicting shorter delay. The speed of onset of symptoms also influenced delay. Patients who experienced a slower onset with prodromal heart related symptoms had longer delay times (mean $1=2.45(2.41)$ hours $v$ mean $2=10.80$ (17.75) hours; $t=3.15 ; \mathrm{df}=87 ; \mathrm{p}<0.05)$.

In order to test the hypothesis that greater delay would be found in those who reported a definite mismatch between symptom expectations and experience, participants were split into two groups. The first group comprised 51 patients (58\%) who reported that the symptoms they experienced during the acute event did not match their expectations of what a heart attack would be like. The second group comprised 26 patients $(29.5 \%)$ who reported that the symptoms they experienced were the same or similar to those expected, together with 11 patients $(12.5 \%)$ who said they could not remember their expectations or did not have any. Longer delays were found for patients whose symptoms did not match their prior expectations of what a heart attack would be like (mean $1=9.31$ (4.53) hours $v$ mean $2=4.53$ (8.88) hours; $t=1.72 ; \mathrm{p}<0.05$ ). Patients were significantly more likely to report a mismatch between expectations and experience of a heart attack if they had not experienced chest pain $\left(\chi^{2}=18.01 ; p<0.01\right)$, and if they had experienced a slow onset of myocardial infarction with prodromal heart related symptoms over the days before the acute event $\left(\chi^{2}=7.95 ; \mathrm{df}=1 ; \mathrm{p}<0.01\right)$.

\section{SYMPTOM EXPERIENCES AND HELP SEEKING} BEHAVIOUR

A relation was also found between symptom experience and expectations and who made the decision to call for help. The decision to call for medical help was more likely to be made by a third party if the patients' experience of symp-

Table 1 Experience of cardiac symptoms and delay in reaching hospital

\begin{tabular}{lllll}
\hline & $\begin{array}{l}\text { Patients reporting } \\
\text { symptom(s) }\end{array}$ & $\begin{array}{l}\text { Mean delay for patients } \\
\text { experiencing the symptom(s) }\end{array}$ & $\begin{array}{l}\text { Mean delay for those not } \\
\text { experiencing the symptom(s) } \\
(\mathrm{h})\end{array}$ & $11.51(20.16)$ \\
\hline $\begin{array}{l}\text { Chest pain } \\
\begin{array}{l}\text { Chest pain and radiating } \\
\text { arm/shoulder/neck pain }\end{array}\end{array}$ & $56(64 \%)$ & $4.89(8.50)$ & $9.70(17.58)$ & $2.15^{\star}$ \\
$\begin{array}{l}\text { Chest pain and radiating } \\
\text { arm/shoulder/neck pain and } \\
\text { collapse }\end{array}$ & $77(42 \%)$ & $3.99(6.03)$ & $7.73(14.68)$ & $2.15^{\star}$ \\
\hline
\end{tabular}

Values are $\mathrm{n}(\%)$ or mean (SD).

${ }^{\star} \mathrm{p}<0.05$. 
toms did not match their prior expectations of a heart attack $\left(\chi^{2}=4.5, \mathrm{df}=1, \mathrm{p}<0.05\right)$. Patients were more likely to make the decision that medical treatment was necessary if they experienced chest pain $\left(\chi^{2}=7.9 ; \mathrm{df}=1\right.$, $\mathrm{p}<0.01)$.

\section{Discussion}

These findings suggest that the experience and interpretation of symptoms may be an important source of delay in arrival at hospital during acute myocardial infarction. Patients tended to reach hospital sooner if they experienced central chest pain, and if their symptoms were of rapid onset and matched prior expectations of what a heart attack would be like. Furthermore, patients whose symptoms matched prior expectations were more likely to call for help themselves rather than rely on others.

These data reinforce the importance of symptom interpretation in relation to help seeking behaviour and delay in reaching hospital. Most patients in our study had prior expectations of the type of symptoms that would be associated with a heart attack. Patients were more likely to recognise the symptoms of acute myocardial infarction if these matched their preconceptions of a heart attack. The typical expectation was one of central chest pain, or radiating pain in the arm neck or shoulder. However, over one third of the sample did not experience central chest pain. These patients were more likely to report that their experience did not match their expectation of what a heart attack would be like and were more likely to show a longer delay to reach hospital.

Delay was more likely when the heart attack presented as a complex range of symptoms compared with a more straightforward "typical" experience of chest pain, radiating pain, or "collapse" (fainting, loss of consciousness, or cardiac arrest). Most patients in our study experienced one or more of a range of symptoms that they might not readily associate with heart problems. These included shortness of breath, chest tightness, nausea and vomiting, fever, and feeling faint, and less than $10 \%$ of the sample associated these symptoms with a heart attack. Thus patients tended to experience a more complex and varied range of symptoms than they had expected. The contrast between this experience and a more simplistic picture of a heart attack as a dramatic event accompanied by central chest pain may have hampered the correct interpretation of their symptoms leading to delay.

The presence of severe chest pain may contribute towards a shorter delay in two ways. First, when the symptoms themselves are severe enough, the physical nature of the heart attack will stimulate the patient to seek help, regardless of any preconceived ideas of symptoms associated with a heart attack. Second, if the symptoms expected are confirmed by experience, then patients' interpretation of the symptoms ("this looks like a heart attack") will encourage a decision to seek help, whereas patients not experiencing severe chest pain may adopt a wait and see approach. However, the lack of uptake of the emergency services when first seeking help was surprising even in those experiencing severe chest pain. Further studies are needed to determine the barriers to contacting emergency services.

Symptom interpretation also influenced who made the decision to call for help, as those patients whose expectations were not fulfilled were less likely to self manage the situation, waiting for someone else to make the decision that medical treatment was necessary. Research suggests that living alone is a risk factor for death following first myocardial infarction. ${ }^{21}$ The reasons for this are unclear but one possibility is that partners intervene to call for help when the patient procrastinates, so avoiding serious delay. It is interesting to speculate that patients whose symptoms did not match prior expectations would have experienced even longer delays had their partner not intervened.

This study has several limitations. Patients' experience and prior expectations of symptoms were elicited retrospectively while they were convalescing in hospital, with the possibility of recall bias and inaccuracy. Moreover, these findings are representative of the population who survived the myocardial infarct, and it is not known how important these factors are in those who have complications, surgical interventions after admission to hospital, or who die before getting to hospital. The study was also limited to those experiencing a first myocardial infarction, and it is possible that patients who have experienced a previous heart attack may have very different expectations of the acute event. A further limitation is that only patients with a confirmed diagnosis of myocardial infarction were included in the study and it is not known how these findings compare with those in patients who are admitted with chest pain or a suspected heart attack. Moreover, we do not know how many of our patients had a vicarious experience of myocardial infarction in a relative, friend, or colleague, or whether this would have influenced symptom interpretation or delay.

Despite its limitations, this study offers a preliminary insight into how the patients' interpretation of their symptoms might contribute to delay in reaching hospital. The data are consistent with clinical observations that many patients experienced their heart attack as a complex array of rather non-specific symptoms and discomfort rather than the " 15 minutes of severe chest pain" which is commonly perceived to be the hallmark of a heart attack. ${ }^{22}$

Recent interventions to reduce mortality from myocardial infarction have focused on educating the public to recognise the symptoms of a heart attack and to seek help early by contacting the emergency services. ${ }^{20} 23$ The findings of the present study reinforce the need for this approach but suggest that further research is needed to include additional symptoms as possible indicators of myocardial infarction and to evaluate the effect of such programmes on patients' ideas of "heart attack" and how they interpret symptoms during the acute event. 
1 Fibrinolytic Therapy Trialists Collaborative Group. Fibrinolytic therapy-indications in suspected acute myocardial lytic therapy - indications in suspected
infarction. Lancet 1994;343:311-22.

2 GISSI. Effectiveness of intravenous thrombolytic treatment in acute myocardial infarction. Lancet 1986;i:397-401.

3 Weaver WD, Cerqueira M, Hallstrom AP, et al. Prehospitalinitiated vs hospital-initiated thrombolytic therapy. The Myocardial Infarction Triage and Intervention Trial. fAMA 1993;270:1211-16

4 Birkhead, J. Time delays in presentation for thrombolytic therapy in 6 district hospitals. BMf 1992;305:445-8.

5 Norris RM. Fatality outside hospital from acute coronary events in three British health districts, 1994-95. BMf 1998;316:1065-70.

6 Maynard C, Weaver WD, Lambrew C, et al. Factors influencing the time to administration of thrombolytic therapy with recombinant tissue plasminogen activator (data from the national registry of myocardial infarction). Am 7 Cardiol 1995;76:548-52.

7 Meischke H, Eisenberg MS, Larsen MP. Prehospital delay interval for patients who use emergency medical services: interval for patients who use emergency medical services:
the effects of heart related medical conditions and the effects of heart related medical conditions and demographic variables. Ann Emerg Med 1993;2:1597-601.
Schroeder JS, Lamb IH, Hu M. The pre-hospital course of patients with chest pain: analysis of the prodromal, symptomatic, decision-making, transportation and emergency room periods. Am $\mathcal{F}$ Med 1978;64:742-7.

9 Dracup K, Moser DK, Eisenberg M, et al. Causes of delay in seeking treatment for heart attack symptoms. Soc Sci Med 1995;40:379-92.

10 Hofgren K, Bondestam E, Johansson FG, et al. Initial pain course and delay to hospital admission in relation to myocardial infarct size. Heart Lung 1988;17:274-80.

11 Schmidt SB, Borsch MA. The prehospital phase of acute MI in the era of thrombolysis. Am f Cardiol 1990;65:1411-15.
12 Clark LT, Bellam SV, Shah AH, et al. Analysis of pre-hospital delay among inner-city patients with symptoms of myocardial infarction: implications for therapeutic intervention. F Natl Med Assoc 1992;84:931-7.

13 Ruston A, Clayton J, Calnan M. Patients' action during their cardiac event: qualitative study exploring differences and modifiable factors. BMF 1998;316:1060-5.

14 Reilly A, Dracup K, Dattolo J. Factors influencing pre-hospital delay in patients experiencing chest pain. Am $\mathcal{F}$ Crit Care 1994;13:350-6.

15 Baumann LJ, Cameron LD, Zimmerman RS, et al. Illness representations and matching labels with symptoms. Health Psychol 1989;8:449-70.

16 Robbins JM, Kirmayer LJ. Attributions of common somatic symptoms. Psychol Med 1991;21:1029-45.

17 Cameron L, Leventhal EA, Leventhal H. Symptom representations and affect as determinants of care seeking in a community-dwelling, adult sample population. Health Psychol 1993;12:171-9.

18 Bishop GD. Lay conceptions of physical symptoms. $\mathcal{A}$ Appl Soc Psychol 1987;17:127-46.

19 Weinman J, Petrie KJ, Moss-Morris R, et al. The illness perception questionnaire: a new method for assessing cognitive representations of illness. Psychol Health 1996;11: cognitive

20 Brighton Heart Attack Study. Heart attack action leaflet. Brighton: BHAS, 1995

21 Case RB, Moss AJ, Case N, et al. Living alone after myocardial infarction. Impact on prognosis. $\mathscr{F} A M A$ 1992;267:51519

22 Treasure T. Pain is not the only feature of a heart attack [letter]. BMF 1998;317:602-3.

23 British Heart Foundation. Information card. London: BHF, 1996.

IMAGES IN CARDIOLOGY

\section{Intermittent mitral valve occlusion by a mobile left atrial thrombus}

A 72 year old woman was admitted for cardiac surgery because of a symptomatic mitral stenosis. She reported intermittent attacks of dyspnoea, increasing over the previous few months. She had an ischaemic cerebral infarction five years ago without any residual effects, and a thrombembolic occlusion of the right femoral artery one month previously, when anticoagulation with coumarin was started. Because of inadequate image quality by transthoracic examination a transoesophageal echocardiography was performed preoperatively. A highly mobile left atrial thrombus could be seen, floating free during systole (A). Diastolic left ventricular inflow leads to an entrapment of the thrombus within the stenotic mitral valve (B) with intermittent reduction of blood flow, as shown by pulse wave Doppler (C). Immediately after examination the patient developed signs of a lung oedema and was operated on as an emergency. The left atrial thrombus was removed and the mitral valve replaced with a $29 \mathrm{~mm}$ mechanical prosthesis. Apart from a transient neurological deficit the postoperative course was uneventful.

This case underlines the necessity of anticoagulation in rheumatic mitral valve disease and demonstrates the typical appearance of a ball shaped thrombus in the left atrium. Owing to an increasingly widespread use of anticoagulation over recent years comparable complications might be prevented in future.
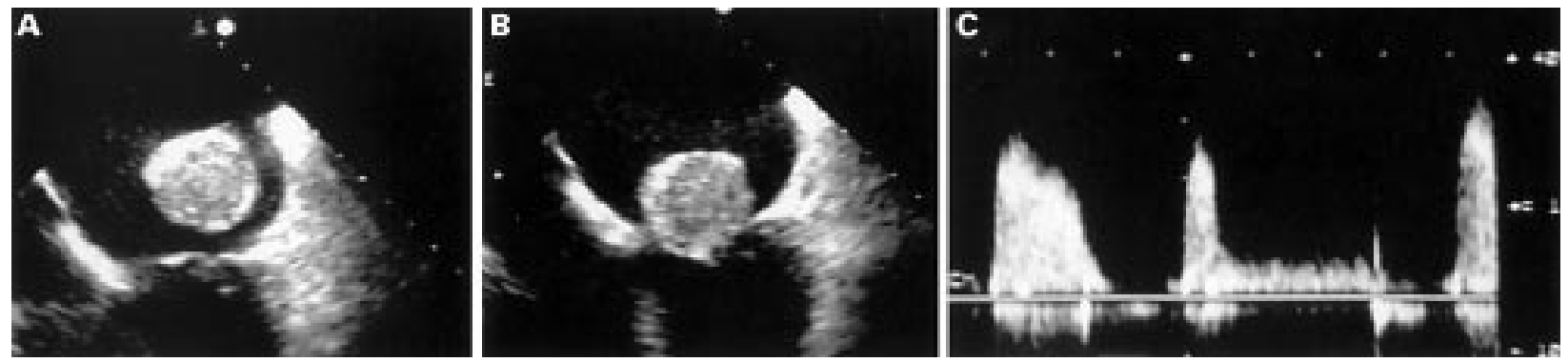